\title{
Estudo epidemiológico sobre as neoplasias malignas da mama no estado do Pará no
}

\section{período de 2013 a 2020}

Epidemiological study on the malignant neoplasms of the breast in the state of Pará in the period

from 2013 to 2020

Estudio epidemiológico sobre las neoplasias malignas de la mama en el estado de Pará en el período de 2013 a 2020

Universidade da Amazônia, Brasil

E-mail: amandaaviz6@gmail.com

Camila de Jesus Prestes da Cruz ORCID: https://orcid.org/0000-0002-7547-6156 Universidade da Amazônia, Brasil

E-mail: camilaprestesbatista@gmail.com Edilene Gemaque Leal ORCID: https://orcid.org/0000-0001-7022-7671 Universidade da Amazônia, Brasil E-mail: lealedilene85@gmail.com

Elaine Samara Pinheiro Mendes da Silva ORCID: https://orcid.org/0000-0003-3101-4758

Universidade da Amazônia, Brasil E-mail: ellaynie1@gmail.com

Jamille Marcelle Ribeiro Costa ORCID: https://orcid.org/0000-0002-4022-6704 Universidade da Amazônia, Brasil E-mail: rjcostamille@gmail.com

Juliane de Jesus Rodrigues Teles ORCID: https://orcid.org/0000-0002-3757-1654 Universidade da Amazônia, Brasil E-mail: telesjuliane1@gmail.com

Keila Ozório Fernandes Barbosa ORCID: https://orcid.org/0000-0002-9093-5736 Universidade da Amazônia, Brasil

E-mail: keilaozorioenfermeira@gmail.com Kodjo Vignonhouandé Jean-Fredich Odilon Nazeba ORCID: https://orcid.org/0000-0001-6795-0586 Universidade da Amazônia, Brasil E-mail: fnazeba@gmail.com

Letícia Lôide Pereira Ribeiro ORCID: https://orcid.org/0000-0002-8784-2547 Universidade da Amazônia, Brasil

E-mail: leticiapereiraloide@gmail.com

Márcia Soraya Quaresma Vera Cruz ORCID: https://orcid.org/0000-0002-0039-755X Universidade da Amazônia, Brasil

E-mail: marciaqveracruz@gmail.com

Maria Gisele Sousa de Sousa

ORCID: https://orcid.org/0000-0002-2712-5482 Centro Universitário Metropolitano da Amazônia, Brasil E-mail: zelysousa@gmail.com

Thalia Otta Ferreira Milanni ORCID: https://orcid.org/0000-0002-7335-2010

Centro universitário do Pará, Brasil E-mail:Thalia_96otta@hotmail.com 


\author{
Rosana de Souza Monteiro \\ ORCID: https://orcid.org/0000-0003-0124-5539 \\ Universidade da Amazônia, Brasil \\ E-mail: monteirosaaa52@gmail.com \\ Wanessa Maiellen Coelho de Oliveira \\ ORCID: https://orcid.org/0000-0002-2480-7277 \\ Universidade da Amazônia, Brasil \\ E-mail: maiellenwanessa@yahoo.com.br
}

\begin{abstract}
Resumo
Objetivo: Identificar e caracterizar os casos diagnosticados de câncer de mama em mulheres do estado do Pará no período de 2013 a 2020. Metodologia: Estudo epidemiológico, descritivo, quantitativo, com dados do Painel Oncologia Brasil, do Departamento de Informática do SUS. As variáveis analisadas foram: ano do diagnóstico, faixa etária, modalidade terapêutica, tempo até início do tratamento e estado de realização do diagnóstico e do tratamento. A análise ocorreu por meio de estatística descritiva no programa Bioestat 5.3. Resultados: Foram diagnosticados 4.387 casos de câncer de mama no período de 2013 a 2020, com prevalência nesse período de 101,22 casos/100 mil mulheres no estado. Foram mais acometidas pela doença as mulheres entre 50 e 59 anos de idade. O tratamento foi realizado sob a forma de quimioterapia para $47 \%$ das pacientes, com início em tempo superior aos 60 dias após diagnóstico para a maioria. O diagnóstico e tratamento foram realizados em instituições do estado de domicílio para parcela expressiva da amostra. Conclusão: O câncer de mama é uma problemática considerável no estado Pará, afligindo um significativo contingente de mulheres. Assim, mais estudos precisam ser realizados para identificar aspectos regionais que influenciam nesse adoecimento e possam embasar medidas estratégicas de saúde a serem tomadas.
\end{abstract}

Palavras-chave: Câncer de mama; Saúde da mulher; Epidemiologia.

\begin{abstract}
Objective: To identify and characterize the diagnosed cases of breast cancer in women in the state of Pará in the period from 2013 to 2020. Method: Epidemiological, descriptive, quantitative study, using data from the Painel Oncologia Brasil, from the SUS Department of Informatics. The variables analyzed were: year of diagnosis, age group, therapeutic modality, time to start of treatment and state of diagnosis and treatment. The analysis took place using descriptive statistics in the software Bioestat 5.3. Results: 4,387 cases of breast cancer were diagnosed in the period from 2013 to 2020, with prevalence of 101.22 cases / 100 thousand women in the state. Women between 50 and 59 years of age were more affected by the disease. The treatment was carried out in the form of chemotherapy for $47 \%$ of the patients, beginning more than 60 days after diagnosis for the majority. Diagnosis and treatment were carried out in institutions in the state of residence for a significant portion of the sample. Conclusion: Breast cancer is a considerable problem in the state of Pará, affecting a significant number of women. Thus, more studies need to be carried out to identify regional aspects that influence this illness and can support strategic health measures to be taken.

Keywords: Breast cancer; Women's health; Epidemiology.

\section{Resumen}

Objetivo: Identificar y caracterizar los casos diagnosticados de cáncer de mama en mujeres del estado de Pará en el período de 2013 a 2020. Metodología: Estudio epidemiológico, descriptivo, cuantitativo, utilizando datos del Painel Oncologia Brasil, del Departamento de Informática del SUS. Las variables analizadas fueron: año de diagnóstico, grupo de edad, modalidad terapéutica, tiempo de inicio del tratamiento y estado del diagnóstico y tratamiento. El análisis se realizó mediante estadística descriptiva en el programa Bioestat 5.3. Resultados: Se diagnosticaron 4,387 casos de cáncer de mama en el período de 2013 a 2020, con una prevalencia en ese período de 101,22 casos/100 mil mujeres en el estado. Las mujeres entre 50 y 59 años fueron las más afectadas por la enfermedad. El tratamiento se realizó en forma de quimioterapia para el $47 \%$ de los pacientes, comenzando más de 60 días después del diagnóstico para la mayoría. El diagnóstico y tratamiento se realizaron en instituciones del estado de residencia para una parte significativa de la muestra. Conclusión: El cáncer de mama es un problema considerable en el estado de Pará, que afecta a un número importante de mujeres. Por lo tanto, es necesario realizar más estudios para identificar los aspectos regionales que influyen en esta enfermedad y pueden apoyar las medidas estratégicas de salud a tomar.
\end{abstract}

Palabras clave: Cáncer de mama; Salud de la mujer; Epidemiología.

\title{
1. Introdução
}

O câncer é uma doença em que há o desenvolvimento descontrolado de algumas células do organismo, que crescem e se multiplicam anormalmente, podendo espalhar-se para outras regiões corporais. Neoplasia maligna e tumor maligno são outros termos utilizados para denominar essa condição, dividida em mais de 100 tipos conhecidos, nomeados de acordo com o órgão ou tecido em que se originou (National Cancer Institute [NCI], 2021). 
O câncer de mama segue esse mesmo padrão de alterações celulares, mas que se originam em tecido mamário. Do ponto de vista morfológico, a mama possui três partes principais: os lobos ou lóbulos, onde se localizam as glândulas mamárias; os ductos, que transportam o leite dos lobos aos mamilos; e o estroma, formado por tecido conjuntivo (fibroso e adiposo) e que envolve toda essa estrutura. São mais comuns as neoplasias malignas que acometem os ductos, chamadas de carcinoma ductal invasivo, e o carcinoma lobular invasivo, originário da porção lobular da mama. Outros cânceres mamários podem ocorrer, porém raramente, dentre esses a doença de Paget e os cânceres de mama dos tipos medular, mucinoso e inflamatório (Centers For Disease Control and Prevention [CDC], 2020).

Vários fatores são apontados como aspectos que contribuem para o aumento do risco de acometimento por essa doença. Dentre esses, destacam-se: a idade, pois há evidências que relacionam maiores níveis de mortalidade por câncer de mama a partir dos 40 anos; a história familiar, sendo o risco aumentado na presença de algum familiar que tenha desenvolvido essa doença; os fatores reprodutivos, representados pela menarca precoce, a menopausa tardia, a idade avançada na primeira gestação e baixa paridade; o estrogênio, principalmente de fonte exógena, como os anticoncepcionais e a terapia de reposição hormonal; e o estilo de vida, relacionado a presença excessiva de gordura na dieta, alcoolismo e tabagismo. Além desses, postula-se também a associação de fatores genéticos à incidência da patologia, sumariamente quanto às mutações dos genes BRCA1 e BRCA2 (Sun et al., 2017).

O diagnóstico das neoplasias mamárias é realizado por meio de várias técnicas disponíveis atualmente. Por um lado há os exames de imagem, representados pela mamografia, ultrassonografia, ressonância magnética e as tomografias computadorizadas (simples, por emissão de pósitrons ou por emissão de fóton único). E também há os exames de biotecnologia molecular, que visam identificar biomarcadores tumorais específicos por meio de técnicas como o sistema de hibridização de ácidos nucleicos, PCR quantitativo de fluorescência em tempo real, biópsia por agulha e imunohistoquímica. Entretanto, ressalta-se que nenhuma técnica isoladamente fornece certeza sobre o quadro da doença, sendo utilizadas para isso várias técnicas de forma associativa (He et al., 2020).

A prevenção tem impacto importante na morbimortalidade pelo câncer de mama. A nível primário, a mudança de estilo de vida, com foco em uma dieta saudável, controle da obesidade, diminuição do consumo de álcool e a prática de atividade física colaboram para redução da exposição a fatores de risco que aumentam a suscetibilidade ao aparecimento da doença. Em termos de prevenção secundária, a realização do autoexame das mamas rotineiramente, bem como de exames de imagem, tem fortes evidências de sua importância no rastreamento precoce e prevenção de agravamento do câncer (Kolak $e t$ al., 2017).

No Brasil, o Instituto Nacional do Câncer estima que no ano de 2020 houve a incidência de 66.280 casos de neoplasia maligna da mama e aproximadamente 18.068 óbitos de mulheres ocasionados por essa patologia. Os fatores genéticos, o histórico reprodutivo e hormonal e os determinantes ambientais são tidos como os principais aspectos que desencadeiam e impulsionam o acometimento pela doença. Por ser um país com grande extensão territorial e, associado a isso, notáveis diferenças de estrutura e acessibilidade aos serviços de saúde, tornam a incidência da doença demasiado variável para as distintas regiões e estados brasileiros (Instituto Nacional do Câncer [INCA], 2021).

Diante disso, o objetivo desse artigo foi realizar um levantamento de modo a identificar os casos diagnosticados de câncer de mama em mulheres no estado do Pará no período de 2013 a 2020 e caracterizá-los quanto a faixa etária, tipos de tratamentos utilizados, tempo entre diagnóstico e início do tratamento e estado de realização do diagnóstico e tratamento.

\section{Metodologia}

Estudo epidemiológico, descritivo, com abordagem quantitativa, tendo como população amostral indivíduos do sexo feminino residentes no estado do Pará, Brasil, acometidas por neoplasia maligna da mama e diagnosticadas nos anos de 2013 a 
2020. Os dados são provenientes do Sistema de Informação Ambulatorial (SIA), do Sistema de Informação Hospitalar (SIH) e do Sistema de Informações de Câncer (SISCAN), disponibilizados no Painel Oncologia Brasil, do Departamento de Informática do Sistema Único de Saúde (DataSUS) (Brasil, 2021a).

O estado do Pará localiza-se na região norte do Brasil, possui área territorial de $1.245 .870,707$ km² e uma população estimada em 8.690.745 pessoas, das quais 4.334.094 são mulheres (cerca de 49,9\%). O Índice de Desenvolvimento Humano é de 0,646 e apresenta população predominantemente urbana (Instituto Brasileiro de Geografia e estatística [IBGE], 2017).

A coleta de dados ocorreu em abril de 2021, para a qual se considerou os seguintes critérios de inclusão: casos confirmados de neoplasias malignas mamárias femininas; diagnóstico realizado no período 2013 a 2020 ; ser residente no estado do Pará. As variáveis selecionadas foram o ano do diagnóstico, a idade, a modalidade terapêutica, o tempo até início do tratamento e a unidade federativa do diagnóstico e tratamento.

Os dados foram tabulados em planilhas do Microsoft Office Excel® e analisados estatisticamente no programa BioEstat (versão 5.3). Para caracterização da amostra foi realizado uma abordagem estatística descritiva, com uso das frequências absolutas (n) e relativas (\%). A taxa de prevalência para o período foi calculada pela razão entre o número de casos no intervalo de tempo pela população no mesmo período, multiplicado por 100 mil (European Patients's Academy on Therapeutic Innovation [EUPATI], 2021). A taxa de incidência, por sua vez, foi calculada pela divisão dos casos novos em um dado período pela população do mesmo período, multiplicado por 100 mil (EUPATI, 2021). A população considerada para os cálculos das taxas foi a fornecida pelas estimativas demográficas do DataSUS (Brasil, 2021b).

Por ser um estudo com dados secundários, não se fez necessária a apreciação do Comitê de Ética em Pesquisa. Apesar disso, foram levadas em consideração todas as normas preconizadas pelo Conselho Nacional de Saúde no que tangem a pesquisa científica.

\section{Resultados e Discussão}

Durante os anos analisados, foram diagnosticados 4.387 casos de neoplasia maligna da mama, com uma taxa de prevalência de período de 101,22 casos para cada 100 mil mulheres residentes no estado do Pará. A média calculada foi de aproximadamente 548,4 casos por ano, em que a amplitude foi representada pelos anos de 2015 (n=430), com o menor número de casos, e 2019 ( $n=653$ ), que apresentou a maior quantidade de novos casos da doença (Tabela 1).

Tabela 1. Distribuição dos casos diagnosticados, segundo ano de diagnóstico, Pará, Brasil, 2021.

\begin{tabular}{|c|c|c|c|}
\hline Ano & $\mathbf{N}$ & $\%$ & Taxa de incidência* \\
\hline 2013 & 514 & 11,7 & 12,86 \\
\hline 2014 & 475 & 10,8 & 11,73 \\
\hline 2015 & 430 & 9,8 & 10,49 \\
\hline 2016 & 578 & 13,2 & 13,94 \\
\hline 2017 & 545 & 12,4 & 13,00 \\
\hline 2018 & 581 & 13,3 & 13,70 \\
\hline 2019 & 653 & 14,9 & 15,23 \\
\hline 2020 & 611 & 13,9 & 14,09 \\
\hline Total & 4.387 & 100 & $13,16 * *$ \\
\hline
\end{tabular}

Notas: *Para 100 mil mulheres/ **Com base nas médias populacional e de casos do período.

Fonte: Dados da Pesquisa (2021). 
Conforme se pode observar na Tabela 1, o número de casos diagnosticados em quatro anos distintos ficou acima da média para o período estudado. Nota-se que houve uma variação na quantidade de diagnósticos entre os anos de 2013 a 2016. Embora destaque-se o crescimento contínuo observado no triênio 2017-2019, seguido de uma redução pouco expressiva no último ano (aproximadamente 6,4\%). A maior taxa de incidência é a referente ao ano de 2019, em que houve o surgimento e diagnóstico aproximado de 15 casos para cada 100 mil mulheres que residem no estado.

O câncer foi mais frequente entre as mulheres com idade superior a 30 anos e inferior aos 70 anos. Houve predominância pela faixa etária dos 50 aos 59 anos (26,92\% dos casos), embora haja grande prevalência entre a quarta e a sexta décadas de vida (26,9\% e 19,9\% dos casos confirmados, respectivamente). Mulheres com 29 anos de idade ou menos $(2,1 \%)$ e aquelas com idade igual ou superior a 80 anos $(3,3 \%)$ foram consideravelmente menos acometidas pela doença em comparação as estratificadas em outras faixas de idade (Tabela 2). A partir disso, pode se inferir que as medidas de prevenção e rastreio devem realmente concentrar-se às mulheres com idade superior aos 30 anos, haja vista o maior acometimento pela doença nessa fase da vida.

Tabela 2. Número de casos diagnosticados, por faixa etária, Pará, Brasil, 2021.

\begin{tabular}{lcc}
\hline Faixa etária & $\mathbf{N}$ & $\%$ \\
\hline 18 a 29 anos & 92 & 2,1 \\
30 a 34 anos & 152 & 3,5 \\
35 a 39 anos & 325 & 7,4 \\
40 a 44 anos & 483 & 11,0 \\
45 a 49 anos & 695 & 15,8 \\
50 a 54 anos & 630 & 14,4 \\
55 a 59 anos & 551 & 12,6 \\
60 a 64 anos & 520 & 11,8 \\
65 a 69 anos & 355 & 8,1 \\
70 a 74 anos & 266 & 6,1 \\
75 a 79 anos & 174 & 3,9 \\
$\geq 80$ anos & 144 & 3,3 \\
\hline Total & $\mathbf{4 . 3 8 7}$ & $\mathbf{1 0 0}$ \\
\hline
\end{tabular}

Fonte: Dados da Pesquisa (2021).

Em estudo realizado no estado do Ceará, em hospital de referência em oncologia na região nordeste, identificou-se que $68 \%$ das mulheres diagnosticadas com câncer de mama estavam na faixa etária dos 40 aos 49 anos, sendo a média das idades calculada em 43 anos (Souza et al., 2017). Em contrapartida, no município de São Paulo, Magalhães et al. (2017) encontraram maior prevalência da doença nos indivíduos que possuíam de 50 a 69 anos de idade.

De modo semelhante, em Taiwan, durante os anos de 1997 a 2013, foram analisadas 125.253 pacientes com diagnóstico confirmado de câncer de mama. Nessa pesquisa, os autores detectaram que houve um maior acometimento de mulheres por volta dos 52 anos de idade $( \pm 12,21$ anos), com pico entre $65-69$ anos, tal qual identificado nos estudos nacionais antes citados. Outrossim, a taxa de prevalência dessas neoplasias foi de 834,37 casos para 100 mil mulheres no ano de 2013, enquanto que a taxa de incidência foi de 93,00 casos para 100 mil mulheres (Liu et al., 2017). Nota-se então que, ao se estabelecer um paralelo, tanto a taxa de incidência quanto a de prevalência desse tipo de neoplasia possuem uma diferença numericamente considerável, sendo mais elevadas se comparadas às taxas identificadas na população feminina paraense. 
De modo geral, esses estudos corroboram os achados relacionados ao estado do Pará dispostos nesta pesquisa, em que houve maior número de casos nesse intervalo dos 40 aos 69 anos de idade.

O tratamento das neoplasias malignas mamárias deve ser realizado em instituições específicas para o manejo terapêutico oncológico, com equipe multiprofissional composta por especialistas na área, para que assim tenham-se resultados aumentados no tocante à sobrevida livre da doença, à melhora da qualidade de vida e aos desfechos funcionais das pacientes (Cardoso et al., 2019). A quimioterapia é uma das opções terapêuticas que podem ser utilizadas no manejo da doença e a ela vem sendo somados grandes avanços nos últimos anos, que contribuem demasiadamente na redução da mortalidade por esse câncer (Tong et al., 2018).

De acordo com os dados analisados na pesquisa, os tratamentos utilizados na abordagem ao câncer de mama consistiram em quimioterapia, radioterapia e cirurgia. A quimioterapia foi a modalidade terapêtica mais empregada em relação às demais, cujo percentual foi equivalente a 47,0\%. Indivíduos submetidos a procedimento cirúrgico, como a exérese da área afetada pelas alterações teciduais neoplásicas, foram um total de 1.860 , o que equivale a $42,4 \%$ da amostra considerada. No mesmo cenário, a modalidade radioterápica foi utilizada por $5,7 \%$ dos casos, configurando-a como uma abordagem empregada de forma bastante restrita no processo terapêutico das neoplasias malignas da mama (Figura 1).

Figura 1. Distribuição dos casos diagnosticados, por modalidade terapêutica empregada, Pará, Brasil, 2021 (N=4.387).

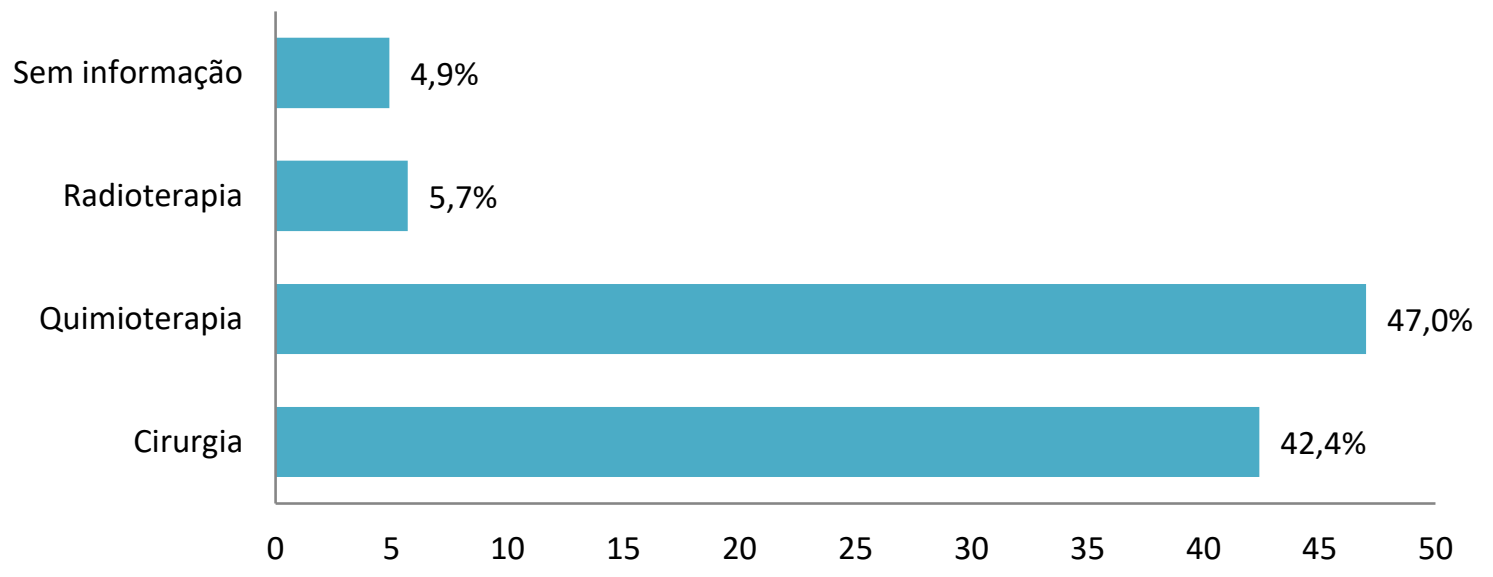

Fonte: Dados da Pesquisa (2021).

Azevedo et al. (2017) encontraram preponderância no uso da quimioterapia em detrimento de outras modalidades terapêuticas na cidade de Goiânia, no período de 2008 a 2012. Segundo os autores, foram 2.160 autorizações para o procedimento, número expressivamente mais alto que o relacionado à radioterapia $(\mathrm{n}=50)$. Silva et al. (2020) identificaram, entre usuárias de serviços prestados por instituição de atendimento oncológico no estado de Sergipe, que todas as mulheres consideradas na amostra foram submetidas à quimioterapia. Na mesma pesquisa, 86,4\% das participantes passaram por procedimento cirúrgico, principalmente para remoção total do tecido mamário. Cerca de 36,0\% realizou radioterapia, o que se alinha aos achados do estado do Pará, em que uma pequena parcela realizou tratamento por essa abordagem. De todo modo, pode-se entender que houve associação das formas de tratamento, com destaque para a quimioterapia e a cirurgia. Entretanto, à luz dos dados coletados para este estudo, não há possibilidade de cruzamento de informações para identificar se houve associação de dois ou mais métodos terapêuticos.

O intervalo entre o diagnóstico e o início do tratamento também pôde ser avaliado. Os dados demonstram que o tratamento se iniciou em até 30 dias para 26,7\% dos casos. Apesar disso, 20,9\% foram iniciados entre 121 e 300 dias, um 
intervalo de tempo consideravelmente longo após a confirmação da patologia. De um modo geral, mais de $64 \%$ dos casos teve sua abordagem terapêutica até os 120 dias após a definição diagnóstica. Aqueles indivíduos com início de tratamento em tempo superior a dois anos somaram apenas $2 \%$ (Tabela 5).

Figura 2. Distribuição dos casos de acordo com o intervalo de tempo entre diagnóstico e início do tratamento, Pará, Brasil, $2021(\mathrm{~N}=4.387)$.

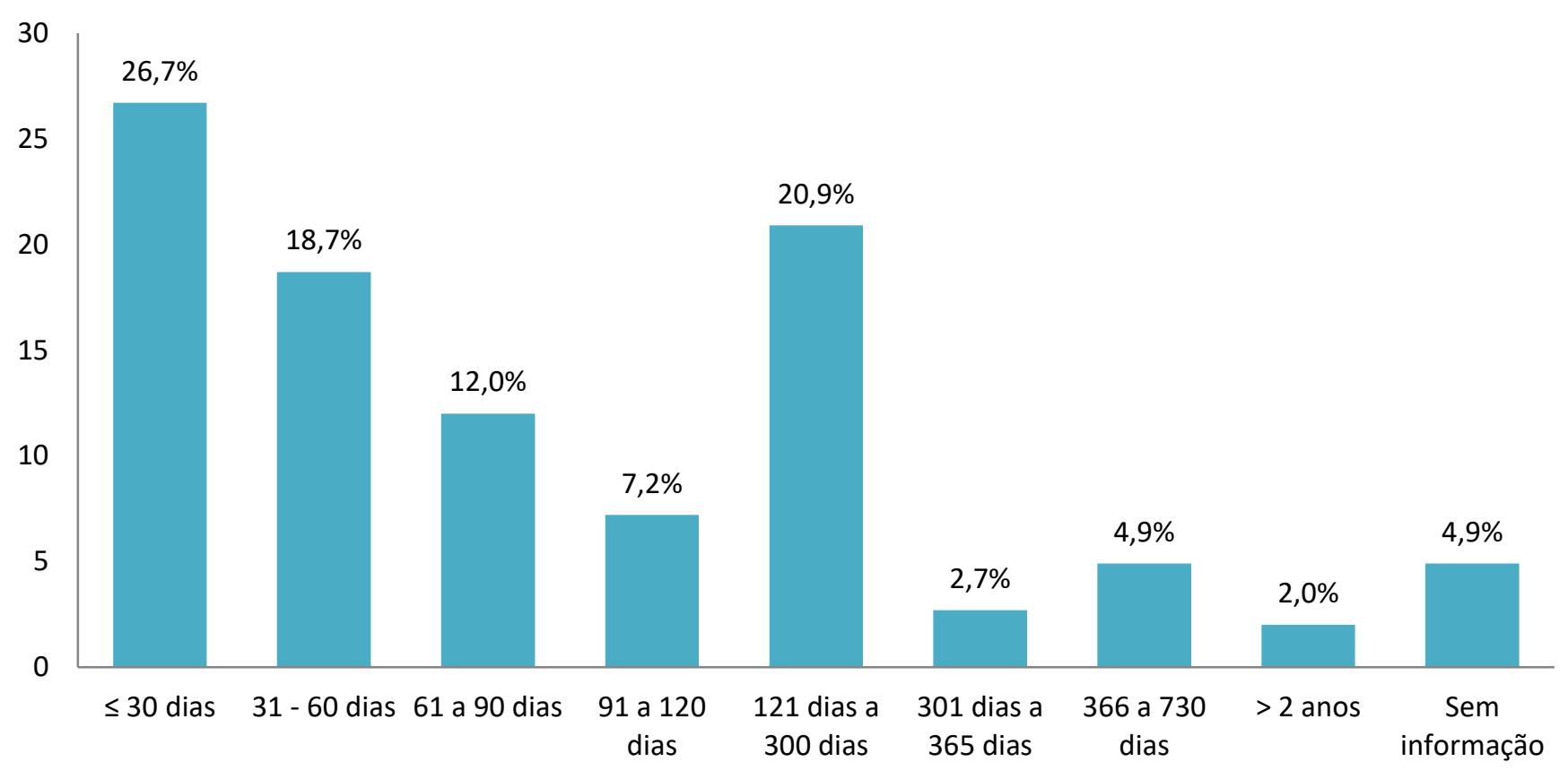

Fonte: Dados da Pesquisa (2021).

Em pesquisa de Constâncio et al. (2020), o período decorrente entre o diagnóstico e o início do tratamento foi de até 60 dias para $64,5 \%$ das participantes entrevistadas e para 35,5\% foi um intervalo superior a esse período. Contrariamente, em outro estudo constatou-se que a maioria $(71,6 \%)$ das mulheres acometidas por câncer de mama só iniciou o tratamento após 60 dias decorridos da confirmação diagnóstica, e em qual somente $28,4 \%$ começou as intervenções em intervalo inferior aos 60 dias (Sousa et al., 2019).

Medeiros et al. (2019) afirmam que mesmo com o estabelecimento da "Lei dos 60 dias" (Lei no 12.732 do Ministério da Saúde), que determina o início do primeiro tratamento de pacientes oncológicos em no máximo 60 dias após diagnóstico, há atraso importante nesse processo. Para esses autores e para Cabral et al. (2019) aspectos tangentes às condições sociodemográficas, características clínicas e do tratamento a ser empregado são os principais fatores que influenciam essa demora na intervenção terapêutica às pacientes. Esse retardamento no início do tratamento acarreta desfechos negativos ao paciente com câncer, visto que haverá desenvolvimento da doença e, por consequência, a utilização de abordagens terapêuticas múltiplas concomitantemente e de forma mais agressiva, além da diminuição das chances de cura e taxa de sobrevida, com prejuízos notórios à qualidade de vida (Carlos Leite, Ruhnke \& Valejo, 2021).

Com foco em identificar qual a situação, principalmente quanto à capacidade, de realização do diagnóstico e do tratamento oncológicos para essa patologia no território estadual estudado, analisaram-se os valores numéricos detectados referentes a essas variáveis. De forma geral, a taxa de diagnósticos feitos nas instituições do próprio estado do Pará compreende a maior parcela $(93,3 \%)$, desvelando que há uma estrutura satisfatória para a execução dos procedimentos necessários para a confirmação da doença. Os estados de São Paulo e Tocantins, respectivamente, são aqueles em que há 
números significativos de participação no processo diagnóstico em parcela da população amostral. Outras unidades federativas também foram identificadas como locais externos de execução diagnóstica, embora com números estatisticamente pouco expressivos.

Tabela 3. Distribuição dos casos identificados, segundo unidade federativa de realização do diagnóstico e tratamento, Pará, Brasil, 2021.

\begin{tabular}{lcccc}
\hline \multirow{2}{*}{ UF } & \multicolumn{2}{c}{ Diagnóstico } & \multicolumn{2}{c}{ Tratamento } \\
\cline { 2 - 5 } & $\mathbf{N}$ & $\mathbf{\%}$ & $\mathbf{N}$ & $\mathbf{\%}$ \\
\hline Pará & 4.095 & 93,3 & 3.901 & 86,4 \\
São Paulo & 113 & 2,6 & 101 & 2,8 \\
Tocantins & 61 & 1,4 & 57 & 1,6 \\
Outros & 118 & 2,7 & 328 & 9,2 \\
\hline Total & $\mathbf{4 . 3 8 7}$ & $\mathbf{1 0 0 , 0}$ & $\mathbf{4 . 3 8 7}$ & $\mathbf{1 0 0}$ \\
\hline
\end{tabular}

Fonte: Dados da Pesquisa (2021).

Semelhante aos números referentes ao diagnóstico, o tratamento também é realizado em sua maioria no mesmo estado de residência dos indivíduos acometidos pela neoplasia, contudo, com índice consideravelmente inferior (86,4\%). Com isso, infere-se que os recursos estaduais utilizados na abordagem terapêutica aos pacientes conseguem suprir a demanda, embora não em sua totalidade, possivelmente devido à eventualidades no manejo da doença que necessitem de modalidades terapêuticas restritas a centros mais especializados.

Os deslocamentos de usuários dos serviços de saúde são esperados em um sistema hierarquizado como o brasileiro, onde há um fluxo de indivíduos principalmente para as unidades de média e alta complexidade. No caso específico do cancer de mama, é evidenciado que aproximadamente 51,3\% dos pacientes não conseguem ser atendidos em seu município de residência, havendo a necessidade de deslocamento para outras localidades para ter acesso à assistência à saúde (Saldanha et al., 2019).

Apesar de avanços, ainda há um grande contingente de deslocamentos de pacientes para outras unidades da federação. As regiões Norte e Nordeste se destacam quanto a proporção de assistência à saúde fora do local de domicílio, sendo os indivíduos do Norte aqueles que maior distância percorrida em busca de atendimento específico para as neoplasias malignas das mamas. O fluxo dessas regiões tem direcionamento orientado principalmente para centros de referência oncológica na região sudeste do Brasil (Souza et al., 2020).

\section{Considerações Finais}

O câncer de mama é um problema considerável no estado do Pará, que afeta principalmente mulheres a partir dos trinta anos de idade e que apresenta uma média anual superior a 500 casos no período analisado. Ademais, apesar do que é determinado pela legislação vigente no que concerne à terapêutica oncológica, grande parte das mulheres só inicia seu tratamento após os 60 dias desde o diagnóstico. Somado a isso, identificou-se que uma pequena, mas considerável, parcela das pacientes precisam se deslocar para outros estados para fins de diagnóstico e/ou tratamento.

Os resultados obtidos com o estudo possibilitaram o conhecimento da situação epidemiológica do câncer de mama no estado, sob alguns aspectos, trazendo evidências sobre o impacto dessa doença na população. Dessa forma, compreender a epidemiologia do agravo em âmbito regional é de sumária importância, visto que desvela informações que podem subsidiar a adoção de estratégias de prevenção, controle e reabilitação orientadas às mulheres acometidas pela doença. Desse modo, é 
possível compreender a importância desta pesquisa não somente para a comunidade científica, mas para os gestores e profissionais relacionados à área da saúde, pois identifica e contextualiza dados imprescindíveis ao entendimento da dinâmica de saúde regional.

Contudo, são necessários estudos mais aprofundados sobre os fatores locais que aumentam os índices de adoecimento desses indivíduos e, também, acerca do atraso no início do tratamento, de modo a contribuir com o conhecimento e possibilitar a formulação de estratégias com intuito de mitigar as condições que favorecem o desenvolvimento e agravamento da patologia, promovendo avanços no prognóstico e qualidade de vida das mulheres.

\section{Referências}

Azevedo, D. B., Moreira, J. C., Gouveia, P. A., Tobias, G. C., \& Morais Neto, O. L. (2017). Perfil das mulheres com câncer de mama. Rev enferm UFPE on line, 11(6), 2264-72.

Brasil. (2021a). Painel-Oncologia Brasil. http://tabnet.datasus.gov.br/cgi/dhdat.exe?PAINEL_ONCO/PAINEL_ONCOLOGIABR.def

Brasil. (2021b). População residente: estudo de estimativas populacionais por município, idade e sexo, $2000-2020$ - Brasil. http://tabnet.datasus.gov.br/cgi/tabcgi.exe?popsvs/cnv/popbr.def

Cabral, A. L. L. V., Giatti, L., Casale, C., \& Cherchiglia, M. L. (2019). Vulnerabilidade social e câncer de mama: diferenciais no intervalo entre o diagnóstico e o tratamento em mulheres de diferentes perfis sociodemográficos. Ciência \& Saúde Coletiva, 24(2), 613-622.

Cardoso, F., Kyriakides, S., Ohno, S., Penault-Llorca, F., Poortmans, P., Rubio, I. T., Zackrisson, S., Senkus, E., \& ESMO Guidelines Committee.(2019). Early breast cancer: ESMO Clinical Practice Guidelines for diagnosis, treatment and follow-up. Annals of oncology: official journal of the European Society for Medical Oncology, 30(8), 1194-1220.

Carlos Leite, G., Ruhnke, B. F., \& Valejo, F. A. M. (2021). Correlação entre tempo de diagnóstico, tratamento e sobrevida em pacientes com câncer de mama: uma revisão de literatura. Colloquium Vitae, 13(1), 12-16.

Centers for Disease Control and Prevention. (2020). What Is Breast Cancer?. https://www.cdc.gov/cancer/breast/basic_info/what-is-breast-cancer.htm

Constâncio, T. B., Gottems, L. B. D., Araújo, S. L., \& Barros, A. F. (2020). Tempo entre diagnóstico e tratamento do câncer de mama no Distrito Federal e fatores associados. Comunicação em Ciências da Saúde, 31(1), 179-187.

European Patients's Academy on Therapeutic Innovation. (2021). Epidemiologic concepts: incidence and prevalence. https://toolbox.eupati.eu/resources/epidemiologic-concepts-incidence-and-prevalence/

He, Z., Chen, Z., Tan, M., Elingarami, S., Liu, Y., Li, T., Deng, Y., He, N., Li, S., Fu, J., \& Li, W. (2020). A review on methods for diagnosis of breast cancer cells and tissues. Cell proliferation, 53(7), e12822.

Instituto Nacional do Cancêr. (2021). Tipos de câncer: câncer de mama. https://www.inca.gov.br/tipos-de-cancer/cancer-de-mama

Instituto Brasileiro de Geografia e Estatística. (2017). Cidades e estados. https://cidades.ibge.gov.br/brasil/pa/panorama

Kameo, S. Y., Barbosa-Lima, R., Ramos, M. J. O., Fonseca, T. V., Vassilievitch, A. C., Costa, J. dos S., Santos, J. C. de O., Santos, D. K. da C., Amorim, B. F., Marinho, P. M. L., Sawada, N. O., \& Silva, G. M. (2021). Perfil clínico-epidemiológico de mulheres em tratamento oncológico de carcinoma ductal invasivo da mama. Research, Society and Development, 10(1), e39110111836.

Kolak, A., Kamińska, M., Sygit, K., Budny, A., Surdyka, D., Kukiełka-Budny, B., \& Burdan, F. (2017). Primary and secondary prevention of breast cancer. Ann Agric Environ Med., 24(4), 549-553.

Liu, F. C., Lin, H. T., Kuo, C. F., See, L. C., Chiou, M. J., \& Yu, H. P. (2017). Epidemiology and survival outcome of breast cancer in a nationwide study. Oncotarget, 8(10), 16939-16950.

Magalhães, G., Brandão-Souza, C., Fustinoni, S. M., Matos, J. C., \& Schirmer, J. (2017). Perfil clínico, sociodemográfico e epidemiológico da mulher com câncer de mama. Rev Fund Care Online, 9(2), 473-479.

Medeiros, G. C., Teodózio, C. G. C., Fabro, E. A. N., Aguiar, S. S., Lopes, A. H. M., Conte, B. C., Silva, E. V., Coelho, L. L. P., Muniz, N. F., Schuab, S. I. P. C., Bergmann, A., \& Thuler, L. C. S. (2020). Fatores Associados ao Atraso entre o Diagnóstico e o Início do Tratamento de Câncer de Mama: um Estudo de Coorte com 204.130 Casos no Brasil. Revista Brasileira De Cancerologia, 66(3), e-09979.

National Cancer Institute. (2021). What Is Cancer?. https://www.cancer.gov/about-cancer/understanding/what-is-cancer

Saldanha, R. F., Xavier, D. R., Carnavalli, K. M., Lerner, K., \& Barcellos, C. (2019). Estudo de análise de rede do fluxo de pacientes de câncer de mama no Brasil entre 2014 e 2016. Cadernos de Saúde Pública, 35(7), e00090918.

Sousa, S. M. M. T., Carvalho, M. G. F. M., Santos Júnior, L. A., \& Mariano, S. B. C. (2019). Acesso ao tratamento da mulher com câncer de mama. Saúde em Debate, 43(122), 727-741.

Souza, B. C., Figueiredo, F. W. S., Sousa, L. V. A., Maciel, E. S., \& Adami, F. (2020). Disparidades regionais no fluxo de acesso às internações por câncer de mama no Brasil em 2004 e 2014. BMC Women's Health, 20(137). 
Research, Society and Development, v. 10, n. 7, e38110716669, 2021

(CC BY 4.0) | ISSN 2525-3409 | DOI: http://dx.doi.org/10.33448/rsd-v10i7.16669

Souza, N. H. A., Falcão, L. M. N., Nour, G. F. A., Brito, J. O., Castro, M. M., \& Oliveira, M. S. (2017). Câncer de mama em mulheres jovens: estudo epidemiológico no nordeste brasileiro. SANARE, 16(2), 60-67.

Sun, Y. S., Zhao, Z., Yang, Z. N., Xu, F., Lu, H. J., Zhu, Z. Y., Shi, W., Jiang, J., Yao, P. P., \& Zhu, H. P. (2017). Risk Factors and Preventions of Breast Cancer. International journal of biological sciences, 13(11), 1387-1397.

Tong,W. S. C., Wu, M., Cho, W. C. S., \& To, K. K. W. (2018). Recent Advances in the Treatment of Breast Cancer. Frontiers in Oncology, 8(227). 